Report no. 09/05

\title{
How fast do radial basis function interpolants of analytic functions converge?
}

\author{
by \\ Rodrigo B. Platte
}

\begin{abstract}
The question in the title is answered using tools of potential theory. Convergence and divergence rates of interpolants of analytic functions on the unit interval are analyzed. The starting point is a complex variable contour integral formula for the remainder in RBF interpolation. We study a generalized Runge phenomenon and explore how the location of centers and affects convergence. Special attention is given to Gaussian and inverse quadratic radial functions, but some of the results can be extended to other smooth basis functions. Among other things, we prove that, under mild conditions, inverse quadratic RBF interpolants of functions that are analytic inside the strip $|\operatorname{Im}(z)|<(1 / 2 \varepsilon)$, where $\varepsilon$ is the shape parameter, converge exponentially.
\end{abstract}

Oxford University Mathematical Institute

Numerical Analysis Group

24-29 St Giles'

Oxford, England OX1 3LB

November, 2009 


\section{Introduction}

Smooth radial basis function (RBF) interpolants are know to converge exponentially (geometrically) fast to functions on native spaces (see e.g., Madych \& Nelson (1992); Wendland (2005)). But for some basis functions, notably Gaussians, the native spaces are quite small, leaving out many analytic functions - for which convergence could be expected. Moreover, on arbitrary node distributions, smooth RBF approximations suffer from a Runge phenomenon, as pointed out in (Platte \& Driscoll, 2005) and (Fornberg \& Zuev, 2007). In this article, using tools of complex analysis, we explore how convergence rates are affected by singularity locations of the function being interpolated and the node distributions.

As usual, we define an RBF interpolant as

$$
s_{f, N}(x):=\sum_{k=1}^{N} c_{k} \phi_{\varepsilon}\left(\left\|x-x_{j}\right\|\right),
$$

where the coefficients $c_{k}$ are computed such that $s_{f, N}\left(x_{j}\right)=f\left(x_{j}\right)$ for $j=1, \ldots, N$. The nodes $x_{j}$ are called centers and $\varepsilon$ is the shape parameter. We shall focus on three smooth (analytic) choices of radial basis:

$$
\begin{array}{ll}
\phi_{\varepsilon}(r)=\exp \left(-(\varepsilon r)^{2}\right) & \text { Gaussians } \\
\phi_{\varepsilon}(r)=1 /\left(1+(\varepsilon r)^{2}\right) & \text { inverse quadratics } \\
\phi_{\varepsilon}(r)=\sqrt{r+(\varepsilon r)^{2}} & \text { multiquadrics }
\end{array}
$$

For a more complete list of radial functions and their properties we refer to the monographs of Buhmann (2003), Wendland (2005), and Fasshauer (2007).

RBF methods have gained much attention in recent years due to their flexibility in node location and ability to handle data in multiple dimensions and complex geometries. Yet, open questions in the theory of convergence of interpolatory approximations remain even for equally spaced nodes in one dimension. In this article we consider approximations on the unit interval $[-1,1]$ to functions that are analytic in a neighborhood of this interval. In this setting, a result from residue calculus can be used to derive a general error formula in terms of a contour integral in the complex plane, which can then be used to obtain rates of convergence that depend on the singularity locations of the target functions and the node distributions.

The shape parameter plays a crucial role in the quality of the approximations. In (Driscoll \& Fornberg, 2002) it was shown that in the flat limit $(\varepsilon \rightarrow 0)$, smooth RBF interpolants converge to polynomial interpolants on the same nodes. In that limit, therefore, the behavior of the approximations is well understood. In (Platte \& Driscoll, 2005) a connection between polynomials and Gaussian interpolants with equally spaced centers was derived through a change of variable. In that case, even when the shape parameter $\varepsilon$ is driven to infinity, convergence rates can be derived using similar tools to those used in classical approximation theory for polynomials, including analysis of a generalized Runge phenomenon and computation of near-optimal node distributions for interpolation. A practical implication of the Runge phenomenon is that it indicates instability of 
the interpolation process - Lebesgue constants that grow exponentially with $N$. The present work extends some of these results to more general node distributions and other basis functions.

This article is organized as follows. In the next section we summarize convergence results in native spaces of positive definite radial basis for completeness and to set notation. In Section 3 we derive an error formula for linear approximations for analytic functions and explore how contour integrals can be used to bound the error of RBF interpolants. In Section 4 we show that Gaussian RBFs on equally spaced nodes and fixed shape parameter diverge when interpolating the same functions that are in the Runge region of polynomial interpolation. In Section 5 we compute equiconvergence contours for RBFs (inverse quadratics and multiquadrics) on equally spaced and Chebyshev centers. In Section 6 we study the associated Runge phenomenon and in Section 7 explore a family of node distributions that prevent the rapid growth of Lebesgue constants. Section 8 presents some concluding remarks.

\section{Convergence on native spaces of positive definite functions}

General convergence results for RBF approximations on a domain $\Omega \in \mathbb{R}^{d}$ have been derived for functions on native spaces $\mathcal{N}_{\phi_{\varepsilon}}(\Omega)$. For strictly positive definite basis functions, such as Gaussians and inverse quadratics, these spaces can be defined as the completion of the pre-Hilbert space

$$
F_{\phi_{\varepsilon}}(\Omega):=\operatorname{span}\left\{\phi_{\varepsilon}(\|\cdot-y\|): y \in \Omega\right\}
$$

equipped with the inner product

$$
\left(\sum_{k=1}^{N} \alpha_{k} \phi_{\varepsilon}\left(\left\|\cdot-x_{k}\right\|\right), \sum_{j=1}^{N} \beta_{j} \phi_{\varepsilon}\left(\left\|\cdot-x_{j}\right\|\right)\right)_{\phi_{\varepsilon}(\Omega)}:=\sum_{j=1}^{N} \sum_{k=1}^{N} \alpha_{k} \beta_{j} \phi_{\varepsilon}\left(\left\|x_{k}-x_{j}\right\|\right) .
$$

Native spaces for conditionally positive definite basis functions, such as multiquadrics, can be defined in a similar fashion and we refer to (Wendland, 2005) for details. For functions in the native spaces of the basis functions being considered in this article, RBF interpolation has been proved to converge at exponential rates with respect to the fill distance,

$$
h_{N}:=\sup _{x \in \Omega} \min _{j=1, \ldots, N}\left\|x-x_{j}\right\|_{2} .
$$

More specifically, for all data sites $x_{j}$ and sufficiently small $h_{N}$,

$$
\left\|f-s_{f, N}\right\|_{L_{\infty}(\Omega)} \leq \exp \left(-c / h_{N}\right)\|f\|_{\mathcal{N}_{\phi_{\varepsilon}}(\Omega)}
$$

for inverse quadratics ${ }^{1}$ and

$$
\left\|f-s_{f, N}\right\|_{L_{\infty}(\Omega)} \leq \exp \left(-c \log h_{N} / h_{N}\right)\|f\|_{\mathcal{N}_{\phi_{\varepsilon}}(\Omega)}
$$

\footnotetext{
${ }^{1} \mathrm{~A}$ similar error bound also holds for multiquadrics.
} 
for Gaussians, where $\Omega$ is any cube in $\mathbb{R}^{d}$ and $\|\cdot\|_{\mathcal{N}_{\phi_{\varepsilon}}(\Omega)}$ is the norm induced by (2.1) (see e.g. Wendland, 2005).

It is worth pointing out that, by Theorem 10.47 in (Wendland, 2005), $\mathcal{N}_{\phi_{\varepsilon}}(\mathbb{R}) \subset$ $\mathcal{N}_{\phi_{\varepsilon}}([-1,1])$, and $\mathcal{N}_{\phi_{\varepsilon}}(\mathbb{R})$ can be characterized using Fourier transforms,

$$
\mathcal{N}_{\phi_{\varepsilon}}(\mathbb{R}):=\left\{f \in L_{2}(\mathbb{R}) \cap C(\mathbb{R}): \widehat{f} / \sqrt{\widehat{\phi}_{\varepsilon}} \in L_{2}(\mathbb{R})\right\}
$$

where $\widehat{f}$ denotes the Fourier transform of $f$. However, because the Fourier transform of smooth basis functions decay exponentially, many analytic functions are excluded from $\mathcal{N}_{\phi_{\varepsilon}}(\mathbb{R})$ and it is difficult to analytically characterize which of them are in $\mathcal{N}_{\phi_{\varepsilon}}([-1,1])$. Moreover, even if $f$ does not belong to $\mathcal{N}_{\phi_{\varepsilon}}([-1,1])$, RBF interpolants may still converge to $f$ under certain assumptions on node distributions. In the next section we use tools of potential theory to shed light on the convergence of RBF approximations to analytic functions that are not in $\mathcal{N}_{\phi_{\varepsilon}}(\mathbb{R})$ and how node distributions affect convergence rates and stability. In what follows, we shall restrict our attention to interpolation on the interval $[-1,1]$.

\section{An error formula for RBF interpolation of ana- lytic functions}

It is well known that node location plays a crucial role in the success of polynomial interpolation and that convergence or divergence of polynomial interpolants can be explained using potential theory, in which a fundamental ingredient is the Hermite formula for the error (see e.g. Davis, 1975).

Theorem 1 (Hermite, 1878) Suppose $f$ is analytic in a closed simply connected region $R$ and $C$ is a simple, closed, rectifiable curve that lies in $R$ and encloses the interpolation points $x_{j}, j=1, \ldots, N$. Then the remainder of the polynomial interpolation for $f$ at $x$ can be represented as the contour integral

$$
f(x)-P_{f, N}(x)=\frac{w_{N}(x)}{2 \pi i} \int_{C} \frac{f(z)}{w_{N}(z)(z-x)} d z,
$$

where $w_{N}(x)=\Pi_{j=1}^{N}\left(x-x_{j}\right)$.

Hermite's formula can be easily generalized to other linear approximations besides polynomial interpolants. To this end, we consider a general linear approximation of a function $f$ from its data values as

$$
\mathcal{L}_{f, N}(x):=\sum_{j=1}^{N} f\left(x_{j}\right) L_{j}(x) .
$$

In the case of interpolation, $L_{j}$ would be a cardinal function. 
Theorem 2 Suppose $f$ is analytic in a closed simply connected region $R$ and $C$ is a simple, closed, rectifiable curve that lies in $R$ and encloses the interpolation points $x_{j}$, $j=1, \ldots, N$. The error at $x$ in the approximation (3.1) is

$$
f(x)-\mathcal{L}_{f, N}(x)=\frac{1}{2 \pi i} \int_{C} \frac{f(z)}{z-x} r_{N}(z, x) d z,
$$

where

$$
r_{N}(z, x)=1-(z-x) \sum_{j=1}^{N} \frac{L_{j}(x)}{z-x_{j}}
$$

Proof Cauchy's integral formula gives

$$
f(x)=\frac{1}{2 \pi i} \int_{C} \frac{f(z)}{z-x} d z
$$

and using complex residue calculus in (3.1) we have

$$
\mathcal{L}_{f, N}(x)=\frac{1}{2 \pi i} \int_{C} f(z) \sum_{j=1}^{N} \frac{L_{j}(x)}{z-x_{j}} d z .
$$

In the case of polynomial interpolation, $r_{N}(z, x)=w_{N}(x) / w_{N}(z)$. More generally, from (3.3) we can see that $r_{N}(z, x)$ can be interpreted as the relative error at $x$ in approximating the function $1 /(z-x)$ with (3.1). A linear method for analytic functions, therefore, is as good as its ability to handle simple poles in the complex plane. Similar error formulas have been derived for quadrature formulas (see e.g. Donaldson \& Elliott (1972); Weideman \& Trefethen (2007); Trefethen (2008)) and for a hybrid Fourier-Chebyshev method (Platte \& Gelb, 2009). The asymptotic behavior of $r_{N}$ as $N \rightarrow \infty$ in the case of RBF interpolation is the main topic of this article.

In the case of meromorphic functions, one can express the error in terms of residues of $f$. The following corollary shows that, in this case, understanding the behavior of $r_{N}$ as a function of $N$ is crucial for estimating rates of convergence and divergence of approximations.

Corollary 2A Suppose $\lim _{z \rightarrow \infty}|f(z)|=0$ and that $f$ is analytic in the complex plane except for a finite number of poles at $\left\{z_{k}\right\}, k=1, \ldots, m_{p}$. Then the error in the approximation (3.1) is

$$
f(x)-\mathcal{L}_{f, N}(x)=-\sum_{k=1}^{m_{p}} \frac{r_{N}\left(z_{k}, x\right)}{z_{k}-x} \operatorname{Res}\left(f ; z_{k}\right),
$$

where $\operatorname{Res}\left(f ; z_{k}\right)$ is the residue of $f$ at $z_{k}$. 


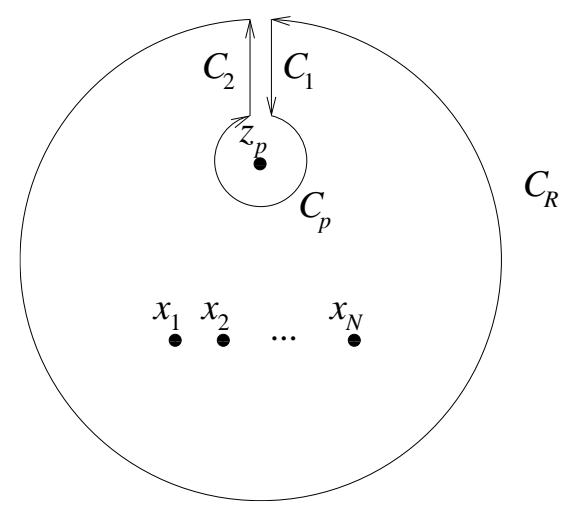

Figure 1: A keyhole contour can be used to prove Corollary 2A, here illustrated with one pole at $z_{p}$.

The decay of the error in Corollary $2 \mathrm{~A}$ as a function of $N$ is given exclusively by the pole locations of $f$ and the function $r_{N}$. The proof of the corollary is sketched in Fig. 1 for the case of one pole. The result follows from Theorem 2 and the use of keyhole contours. Because

$$
\left|\int_{C_{R}} \frac{f(z)}{z-x} r_{N}(z, x) d z\right| \leq 2 \pi R \max _{|z|=R}\left|f(z)\left(\frac{1}{z-x}-\sum_{j=1}^{N} \frac{L_{j}(x)}{z-x_{j}}\right)\right|
$$

and $\lim _{z \rightarrow \infty}|f(z)|=0$, the integral over $C_{R}$ vanishes as its radius $R$ tends to infinity, leaving only integrals around each pole, which are the residues at the poles. We point out that in the case of polynomial interpolation, the requirement on the decay of $f$ at infinity can be replaced with $|f(z)| \leq k\left|z^{N-1}\right|$, for some constant $k$ and sufficiently large $|z|$.

More generally, we have the following uniform error bound on the interval $[-1,1]$. Here we define $\|\cdot\|_{[-1,1]}$ as the supremum norm over $[-1,1]$.

Corollary 2B Under the assumptions of Theorem 2 we have

$$
\left\|f-\mathcal{L}_{f, N}\right\|_{[-1,1]} \leq M e^{N \max _{z \in C} \psi_{N}(z)},
$$

where

$$
\psi_{N}(z)=\frac{1}{N} \max _{x \in[-1,1]} \log \left(\left|r_{N}(z, x)\right|\right)
$$

and

$$
M=\frac{\operatorname{arclength}(C) \max _{z \in C}|f(z)|}{2 \pi \min _{x \in[-1,1], z \in C}|z-x|}
$$

The motivation for the definition of $\psi_{N}(z)$ is the expectation that approximations will converge geometrically with $N$ for sufficiently smooth functions. In fact, for inverse quadratics, convergence results on the native space $\mathcal{N}_{\phi_{\varepsilon}}(\mathbb{R})$ can be used to estimate $\psi_{N}(z)$ 
away from the unit interval. The following theorem combines the theory developed in this section with results for functions on native spaces, and to our knowledge, has not previously appeared in the literature.

Theorem 3 Suppose $f$ is analytic in an open region that includes the strip $|\operatorname{Im}(z)| \leq$ $1 /(2 \varepsilon)$ in its interior. Then for all node distributions $\left\{x_{j}\right\}, j=1, \ldots, N$ with fill distance $h_{N}=O(1 / N)$ on the unit interval, the error in the inverse quadratic RBF interpolation is

$$
\left\|f-S_{N, f}\right\|_{L_{\infty}[-1,1]}=O\left(e^{-c N}\right), \quad \text { as } N \rightarrow \infty,
$$

for some constant $c>0$.

The constant $c$ is estimated in Section 5 for some choices of node distributions and shape parameters.

Proof Let $g_{z}(x)=1 /(z-x)$ with $\operatorname{Im}(z)>0$. The function $r_{N}(z, x)$ is the relative error at a point $x$ in the approximation of $g_{z}(x)$. Taking the Fourier transforms of $g_{z}(x)$ and $\phi_{\varepsilon}(x)=1 /\left(1+(\varepsilon x)^{2}\right)$, we have

$$
\frac{\left|\widehat{g_{z}}\right|}{\sqrt{\widehat{\phi_{\varepsilon}}}}= \begin{cases}\frac{4 \sqrt{\varepsilon}}{\sqrt{\pi}} \exp (\omega(1 / \varepsilon-2 \operatorname{Im}(z))) & \text { if } \omega \leq 0 \\ 0 & \text { if } \omega>0\end{cases}
$$

Therefore, from the convergence results on native spaces summarized in Section 2, we know that $r_{N}(z, x)$ must decay at exponential rates for all $z$ outside the strip $|\operatorname{Im}(z)| \leq 1 /(2 \varepsilon)$ and all $x$ in $[-1,1]$. We now use the error bound in Corollary $2 \mathrm{~B}$ to complete the proof.

Theorem 3 also holds for other smooth basis functions with singularities in the complex plane, such as $\phi_{\varepsilon}(r)=1 /\left(1+(\varepsilon r)^{2}\right)^{2}$ or $\phi_{\varepsilon}(r)=1 /\left(1+(\varepsilon r)^{2}\right)^{4}$. The native space for Gaussians, however, is much smaller and excludes all functions $g_{z}$ because the Fourier transform of a Gaussian is also a Gaussian. In the next section we focus on Gaussian RBFs with equally spaced centers and their relation to polynomials.

\section{Gaussian and polynomial interpolation on equally spaced nodes}

Gaussians are entire functions, in contrast to inverse quadratics or multiquadrics, which have have singularities at $x_{k} \pm i / \varepsilon$ (poles for inverse quadratics and branch points for multiquadrics). While inverse quadratics (and generalizations) are rational functions, Gaussian RBFs can be related to polynomials, especially when the centers are equally spaced.

It is well known that, in polynomial interpolation, if a family of nodes $\left\{x_{j}\right\}, j=$ $1, \ldots, N$, has the property that

$$
\lim _{N \rightarrow \infty}(1 / N) \log \left|w_{N}(z)\right|=\int_{-1}^{1} \mu(x) \log |z-x| d x,
$$



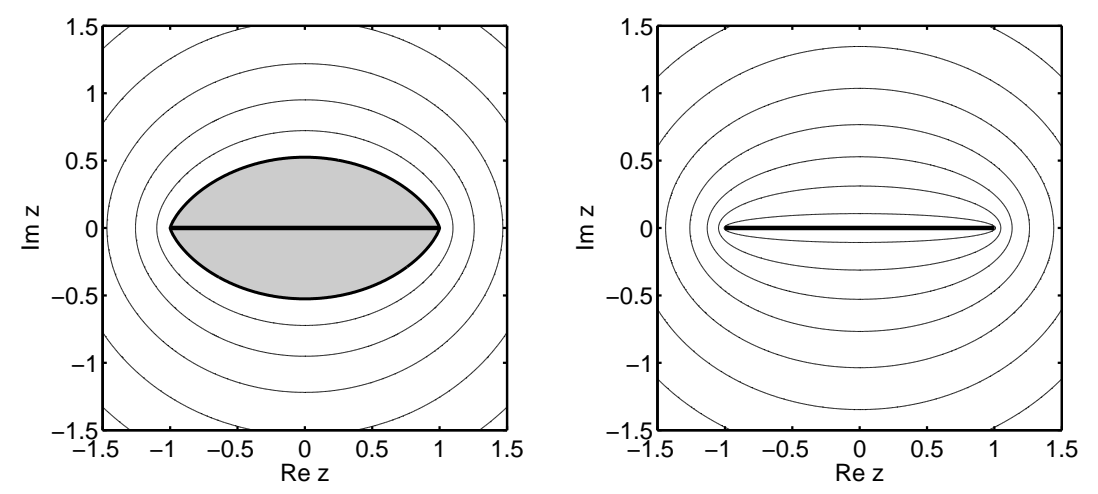

Figure 2: Equiconvergence contours for polynomial interpolation on equispaced nodes (left) and Chebyshev nodes (right).

for some node density function $\mu(x)$ on $[-1,1]$, then

$$
\left|r_{N}(z, x)\right| \rightarrow \exp (N(u(x)-u(z))), \quad \text { as } N \rightarrow \infty,
$$

where $u(z):=\int_{-1}^{1} \mu(x) \log |z-x| d x$ is called the logarithmic potential (see e.g. Davis (1975); Fornberg (1996)).

Combining Corollary 2B with the result above we have that polynomial interpolation converges exponentially whenever $f$ is singular only in regions with $u(x)-u(z)<0$. For node distributions that result in $u(x)-u(z)>0$ in some region outside the unit interval, we know by Corollary 2A, that there are analytic functions on the unit interval for which the interpolation precess is divergent. This phenomenon was famously demonstrated by Runge for polynomial interpolation on equispaced nodes (Runge, 1901) and we shall refer to these regions as Runge regions.

Fig. 2 shows the contour levels of $u$, also known as equiconvergence contours, for two node density functions: $\mu=1 / 2$ and $\mu(x)=1 /\left(\pi \sqrt{1-x^{2}}\right)$. The former density function corresponds to uniform node distributions and the latter to Chebyshev nodes, given by $x_{j}=-\cos [(j-1) \pi /(N-1)]$, or to other roots or extrema of Jacobi polynomials. The left figure shows the contours for uniform node distributions, with the Runge region shown in gray. Chebyshev nodes, on the other hand, are near optimal. The right figure shows that polynomial interpolation is convergent for any function that is analytic in a neighborhood of the unit interval.

Unfortunately, as stated in the next theorem, Gaussian RBFs with equally spaced centers are also susceptible to the Runge phenomenon to almost the same extent that polynomials are. We believe a more general result should hold for other node distributions, but we are only able to prove the theorem for equispaced centers.

Theorem 4 Assume that $x_{j}$ are equally spaced nodes. The interpolation process by Gaussian RBFs with fixed shape parameter is convergent for functions that are analytic inside the Runge region of polynomial interpolation, i.e., the region enclosed by the curve

$$
2 \log 2=\operatorname{Re}((z+1) \log (z+1)-(z-1) \log (z-1)) .
$$



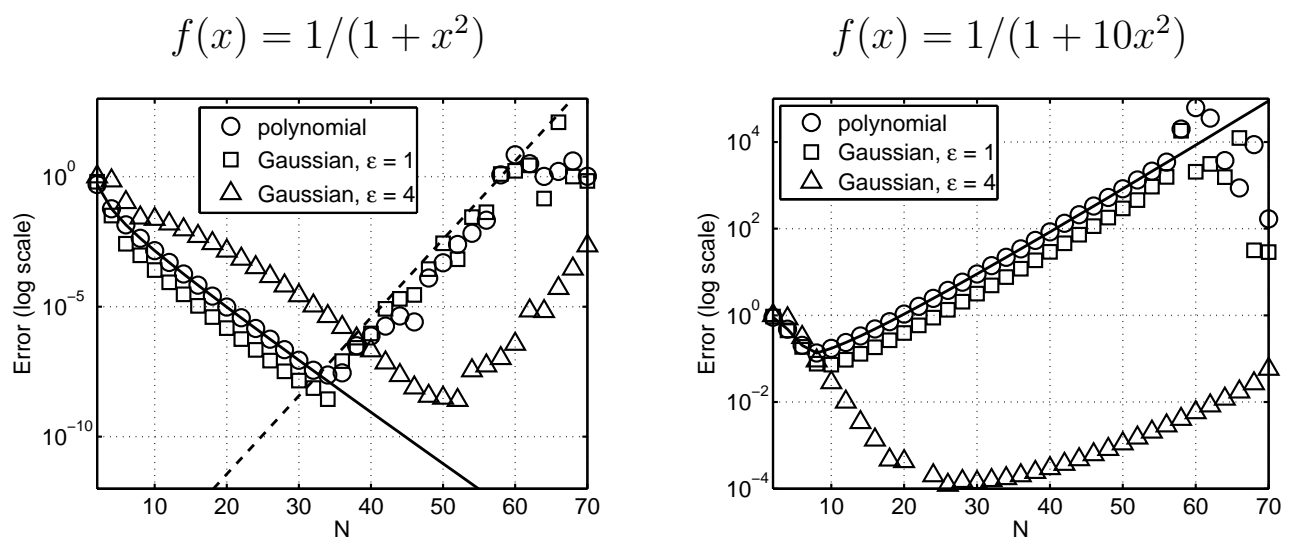

Figure 3: Semilog plot of the maximum error in interpolation by polynomials and Guassian RBFs on equally spaced nodes. The dashed line represents an estimate of the growth of rounding errors in polynomial interpolation due to large Lebesgue constants. The solid lines show the truncation error given by Corollary $2 \mathrm{~A}$ for polynomial interpolation.

Moreover, if polynomial interpolation fails to converge to a target function $f$, so will Gaussian RBF interpolation.

The proof is provided in the Appendix. The theorem precisely defines the set of analytic functions that can be recovered by interpolation on equally spaced points with Gaussian RBFs. For large values of the shape parameter $\varepsilon$ and moderate values of $N$, Gaussian interpolants may differ significantly from polynomial ones, but this difference decreases as $\varepsilon^{2} / N \rightarrow 0$. A consequence of the existence of a Runge region is that the Lebesgue constant (norm of the interpolation operator) must grow exponentially, making the interpolation process unstable.

In Fig. 3 we show the behavior of the $L_{\infty}$-error for polynomial and Gaussian interpolation with $\varepsilon=1$ and $\varepsilon=4$. The left figure presents the error as a function of $N$ for interpolants of $f(x)=1 /\left(1+x^{2}\right)$, which is analytic inside the Runge region and approximations are predicted to converge. The truncation error indeed decreases at exponential rates until rounding errors start to dominate. The Lebesgue constant for polynomial interpolation on equally spaced nodes is $O\left(2^{N} /(n \log n)\right)$ (Turetskii, 1940). As shown in this figure, the norm of the interpolation operator for Gaussian RBFs also grows with similar rates. The error plots on the right frame are for the interpolation of $f(x)=1 /\left(1+10 x^{2}\right)$, which has poles at $\pm i / \sqrt{10}$, and therefore has singularities inside the Runge region. In this case all three curves diverge as predicted. The interpolants were computed using barycentric formulas - see e.g. (Berrut \& Trefethen, 2004) for polynomials and (Platte \& Driscoll, 2005) for Gaussians.

In order to avoid the Runge phenomenon when interpolating with Gaussian RBFs, one may choose to vary the shape parameter $\varepsilon$ with $N$. We conclude this section with a summary of these interpolants according to shape parameter choices:

- $\varepsilon \rightarrow 0$, the flat limit: RBF interpolants are identical to polynomials, unstable 
(Driscoll \& Fornberg, 2002).

- Fixed $\varepsilon$ : asymptotically, as $N \rightarrow \infty$, Gaussains and polynomials have similar convergence properties, unstable (Theorem 4).

- $\varepsilon=O(\sqrt{N})$ : approximations still converge at geometric rates for sufficiently smooth functions but Runge regions still exist in this case, unstable (Platte \& Driscoll, 2005).

- $\varepsilon=O(N)$ : interpolants fail to converge (saturation error), but this regime is of practical interest because it leads to better conditioned interpolation matrices, stable (Boyd, 2009).

\section{Equiconvergence contours}

The focus in this section is the computation of the function $\psi_{N}$ used to bound the error in Corollary 2B. As will be seen, numerical results support the conjecture that for smooth RBFs

$$
\psi_{\infty}(z):=\lim _{N \rightarrow \infty} \psi_{N}(z)=\lim _{N \rightarrow \infty} \sup _{x \in[-1,1]} \frac{1}{N} \log \left|r_{N}(z, x)\right|
$$

is nonzero and bounded almost everywhere in the complex plane. The function $\psi_{\infty}$ provides us with the asymptotic exponential rates of convergence as $N \rightarrow \infty$. In particular, we shall refer to the level curves of $\psi_{\infty}$ as equiconvergence contours. When these contours are closed, with the unit interval in their interior, we can restate Corollary $2 \mathrm{~B}$ as follows.

Corollary 4A Suppose the condition $\psi_{\infty}(z)=-c<0$ defines a simple, closed, rectifiable curve $C$ that encloses the interval $[-1,1]$ in its interior. If $f$ is an analytic function in an open region $R$ that contains $C$ in its interior, then the approximation (3.1) converges uniformly in $[-1,1]$ and

$$
\left\|f-\mathcal{L}_{f, N}\right\|_{[-1,1]}=O\left(e^{-c N}\right) .
$$

This error estimate is a direct consequence of Corollary $2 \mathrm{~B}$ when $\psi_{\infty}(z)$ is constant for all $z \in C$. Unfortunately, because closed formulas for RBF cardinal functions are not known in general, we rely on numerical computations to estimate $\psi_{\infty}(z)$. Before proceeding, we point out some properties of $\psi_{N}$.

Proposition 5 Assume that the interpolation nodes $x_{k}$ are in $[-1,1]$. Then $\psi_{N}$ has the following properties.

1. $\psi_{N}(x) \geq 0$ for all $x \in[-1,1], x \neq x_{k}$.

2. $\lim _{z \rightarrow x_{k}} \psi_{N}(z)=\infty, \forall N<\infty$. 
3. If $\psi_{\infty}(z)>0$ for some $z$ in $\mathbb{C}$, then the Lebesgue constants, $\Lambda_{N}:=\left\|\sum_{k=1}^{N}\left|L_{k}(x)\right|\right\|_{L_{\infty}[-1,1]}$, must grow faster then any power of $N$, indicating that the interpolation process is unstable.

4. $\psi_{\infty}(z)<0$ for all $z$ with $|\operatorname{Im}(z)|>1 /(2 \varepsilon)$ for inverse quadratics (under the assumptions of Theorem 3).

Property 1 follows because $r_{N}(x, x)=1$. The second property holds because, by definition, $r_{N}(z, x)$ has simple poles at $z=x_{k}$, whenever $x \neq x_{k}$. The third property follows from the definition of operator norm and the fact that $\left|S_{f, N}\right|$ grows exponentially for functions with poles at points with $\psi_{\infty}(z)>0$. Property 4 is a consequence of Theorem 3.

Our first set of computations of $\psi_{N}$ is for inverse quadratics with equally spaced centers. Because of Theorem 3, the main focus is on the strip $|\operatorname{Im}(z)|<1 /(2 \varepsilon)$. To avoid rounding errors in the computation of cardinal functions, we use MAPLE, which handles rational expressions efficiently. In this case, we can compute $\psi_{N}$ in exact arithmetic for moderate values of $N$. Some of the computations presented here required the use of interpolation matrices with condition numbers of order $10^{120}$.

Fig. 4 presents the contour levels of $\psi_{N}$ for $N=10,30,50,70,90$. The plots were generated with shape parameter fixed at $\varepsilon=0.5$ and centers at equally spaced points. We observe that the contour curves converge quickly with $N$ as there is little difference between $\psi_{70}$ and $\psi_{90}$. We take $\psi_{90}$ as a good estimate to $\psi_{\infty}$. In all frames we show the pole locations of the basis functions $\left(x_{k} \pm i / \varepsilon\right)$. The zero-level of $\psi_{90}$ is marked with a thick line and $\psi_{90}>0$ in the region enclosed be this curve giving an estimate of the Runge region - by Corollary $2 \mathrm{~A}$ there are functions with poles in the interior of this region for which the error grows exponentially. Another remarkable feature of $\psi_{90}$ is that it is nearly constant outside the strip $|\operatorname{Im}(z)|<1 /(2 \varepsilon)$, indicating that interpolants to all functions included in Theorem 3 converge at the same rate. The value of $\psi_{90}$ outside this strip is approximately -0.79 .

Fig. 5 shows a surface plot of $\psi_{7}$ (7 equally spaced centers). The blowup at the interpolation nodes predicted in Proposition 5 is evident. These singularities, however, tend to be difficult to visualize as $N$ grows (one needs to sample $\psi_{N}(z)$ exactly at $x_{K}$ ) - as illustrated in Fig. 6. The spikes of $\psi_{90}$ are too narrow to be seen. As in polynomial interpolation, we believe that in the limit, $\psi_{\infty}$ is indeed bounded on $[-1,1]$. We also point out the non-smooth change of behavior at the lines $|\operatorname{Im}(z)|=1 /(2 \varepsilon)$, consistent with the contours shown in Fig. 4.

Fig. 7 shows contours for inverse quadratic RBFs with $\varepsilon=1$ and equidistant nodes. The behavior of $\psi_{N}$ in this case is very similar to the previous case - shape parameter $\varepsilon=0.5$. The main differences are that the poles of the basis functions and the strip $|\operatorname{Im}(z)|=1 /(2 \varepsilon)$ are half-way closer to the real line and the magnitude of the values of $\psi_{N}$ is smaller than in the previous case. As expected, as the shape parameter is increased, convergence and divergence rates also decrease. Note that a Runge region is also present in this case, since $\psi_{N}$ takes positive values in a neighborhood of the interval. 
$\psi_{10}$
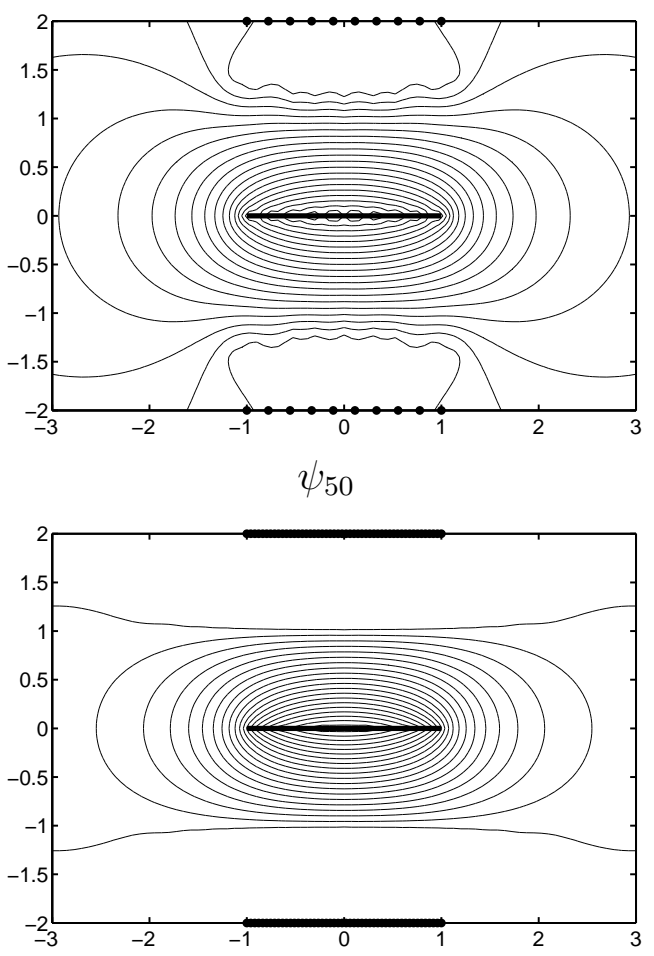

$\psi_{30}$
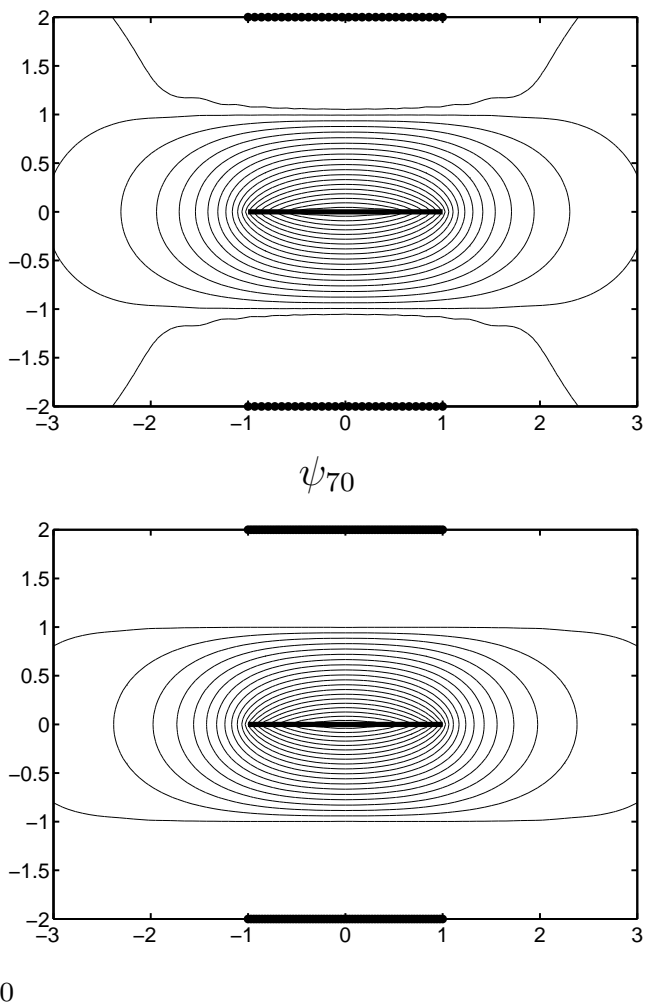

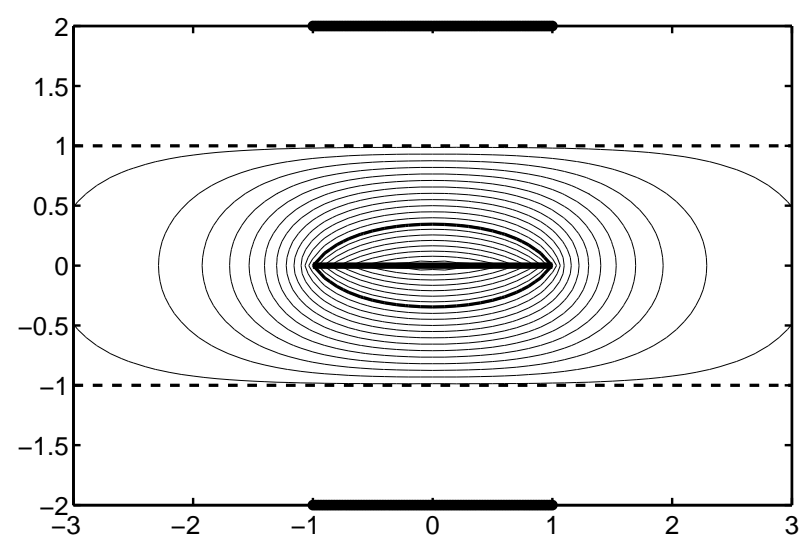

Figure 4: Contour levels of $\psi_{N}$ for inverse quadratic RBFs with equispaced centers and $\varepsilon=0.5$. Twenty contour levels are shown for $N=10,30,50,70,90$. A thick line marks the unit interval and the poles of the basis functions are marked with dots. The last frame also shows the strip bounded by $|\operatorname{Im}(z)|=1 /(2 \varepsilon)$ and the zero-level curve (thick line). Other level values of $\psi_{90}$ shown are $-0.78,-0.72, \ldots, 0.38,0.44$. 


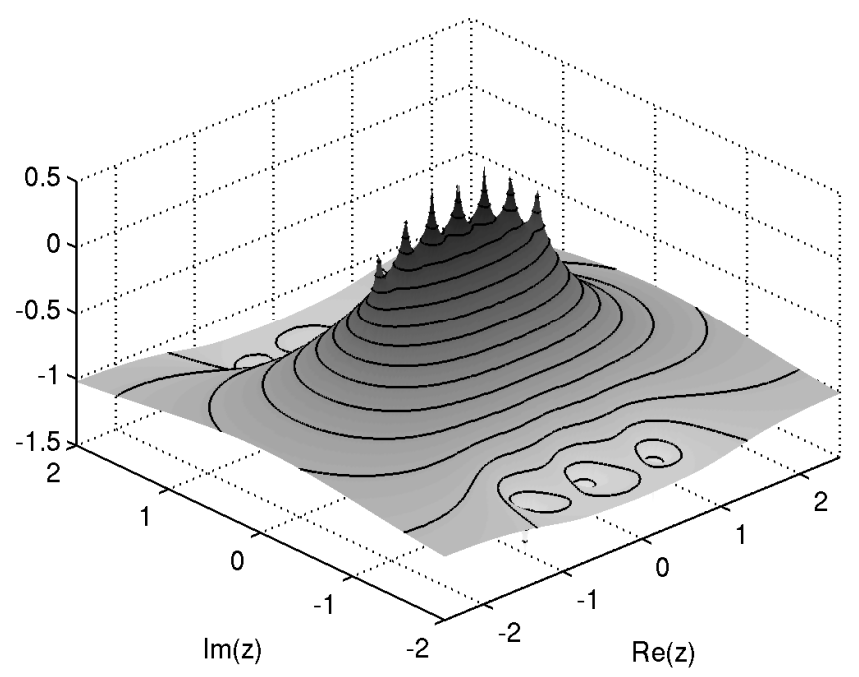

Figure 5: Surface plot of $\psi_{7}$ for $\varepsilon=0.5$ and equispaced nodes. Poles of $\psi_{7}$ can be seen at interpolation points.

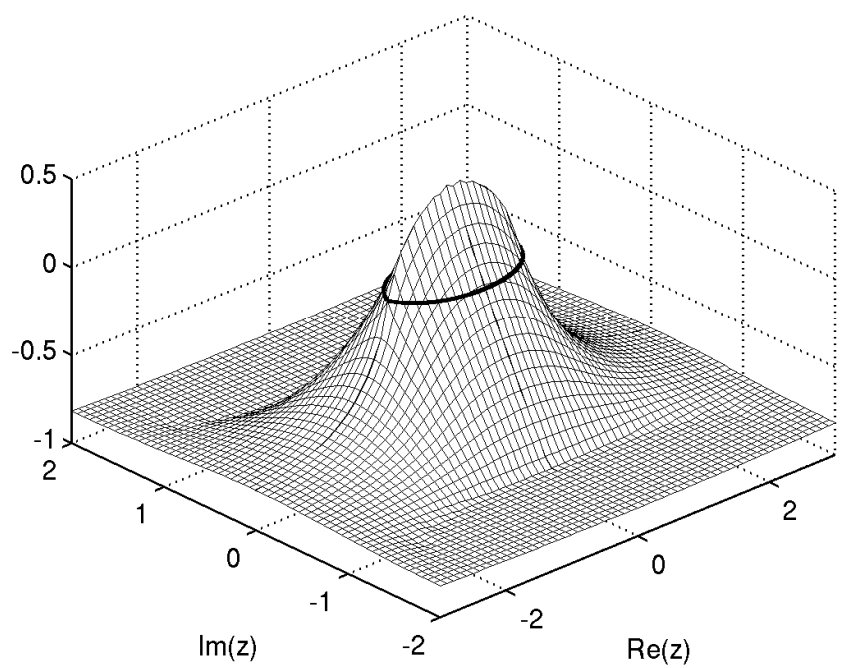

Figure 6: Surface plot of $\psi_{90}$ for $\varepsilon=0.5$ and equispaced nodes. The zero-contour is shown with a thick line - cf. last frame of Fig. 4. Ninety spikes of $\psi_{90}$ in $[-1,1]$ are too narrow to be visible in this plot. 

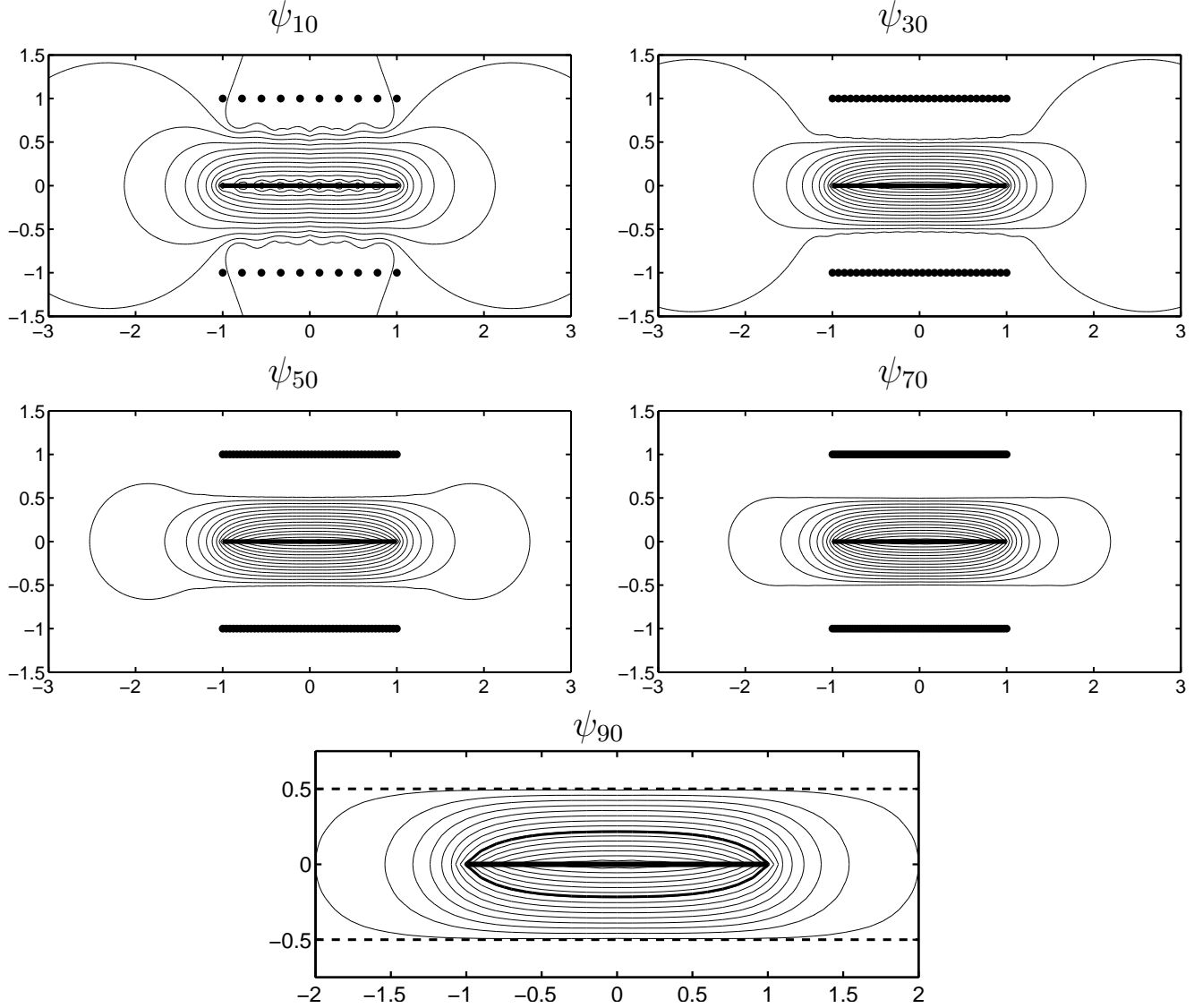

Figure 7: Contour levels of $\psi_{N}$ for inverse quadratic RBFs with equispaced centers and $\varepsilon=1$. Fifteen contour levels are shown for $N=10,30,50,70,90$. A thick line marks the unit interval and the poles of the basis functions are marked with dots. The last frame also shows the strip bounded by $|\operatorname{Im}(z)|=1 /(2 \varepsilon)$ and the zero-level curve (thick line). Level values of $\psi_{90}$ shown are $-0.42,-0.37, \ldots, 0.23,0.28$. 

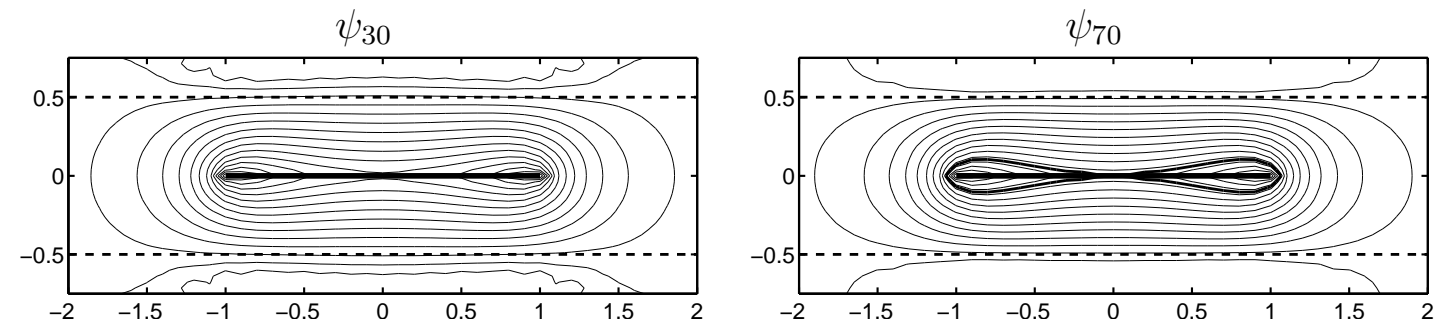

Figure 8: Contour levels of $\psi_{N}$ for inverse quadratic RBFs, Chebyshev nodes, and $\varepsilon=1$. Fifteen contour levels are shown for $N=30$ and $N=70$. A thick line marks the unit interval and dashed lines represent $|\operatorname{Im}(z)|=1 /(2 \varepsilon)$. Level curves in the last frame correspond to $\psi_{70}=-0.56,-0.51, \ldots, 0.14,0.19$. A thick line marks the level zero of $\psi_{70}$.

The regions of positive values of $\psi_{90}$ in Fig. 4 and Fig. 7 indicate that clustering near endpoints of the interval is needed. Chebyshev nodes, however, are overly clustered, as illustrated in Fig. 8. The figure shows the contours of $\psi_{N}$ for inverse quadratic RBFs with centers at Chebyshev points and $\varepsilon=1$. The figure also indicates that $\psi_{\infty}$ takes positive values in a neighborhood of the interval, with the distinction that the Runge region is now around the endpoints of the interval. Functions with singularities near $x= \pm 1$ lead to divergent interpolants. The sharp change of behavior at $|\operatorname{Im}(z)|=1 /(2 \varepsilon)$ is also observed for Chebyshev nodes. The values of the level curves also predict that convergence is faster (and divergence slower) that in the equidistant centers case.

The theory presented in Section 3 (except for Theorem 3) is general and also applicable to other basis functions, such as multiquadrics. The difficulty in this case is the computation of the cardinal functions needed to evaluate $\psi_{N}$. Fig. 9 and Fig. 10 were computed in double precision arithmetic using MATLAB. For multiquadrics with equispaced centers and $\varepsilon=1$, the condition numbers of interpolation matrices exceed $10^{16}$ when $N>23$, making it difficult to estimate the asymptotic behavior of $\psi_{N}$ for large $N$. However, Fig. 9 indicates that the equiconvergence contours are qualitatively similar to those of inverse quadratics for moderate values of $N$. In particular, we observe that a Runge region is likely to be present in the limit $\psi_{\infty}$. A strip similar to the one stated in Theorem 3 seems to emerge, but unfortunately larger values of $N$ would be needed to estimate asymptotic rates of convergence.

\section{The Runge phenomenon}

We now turn our attention to the Runge phenomenon, Lebesgue constants, and their relation to node distributions. Fig. 11 presents Runge regions estimated from computations described in the previous section. Both equidistant and Chebyshev nodes lead to interpolants that are susceptible to the Runge phenomenon. Inverse quadratic RBFs with $\varepsilon=1$ were used to obtain these regions.

The left frame of Fig. 11 (equidistant nodes) also presents singularity locations for 

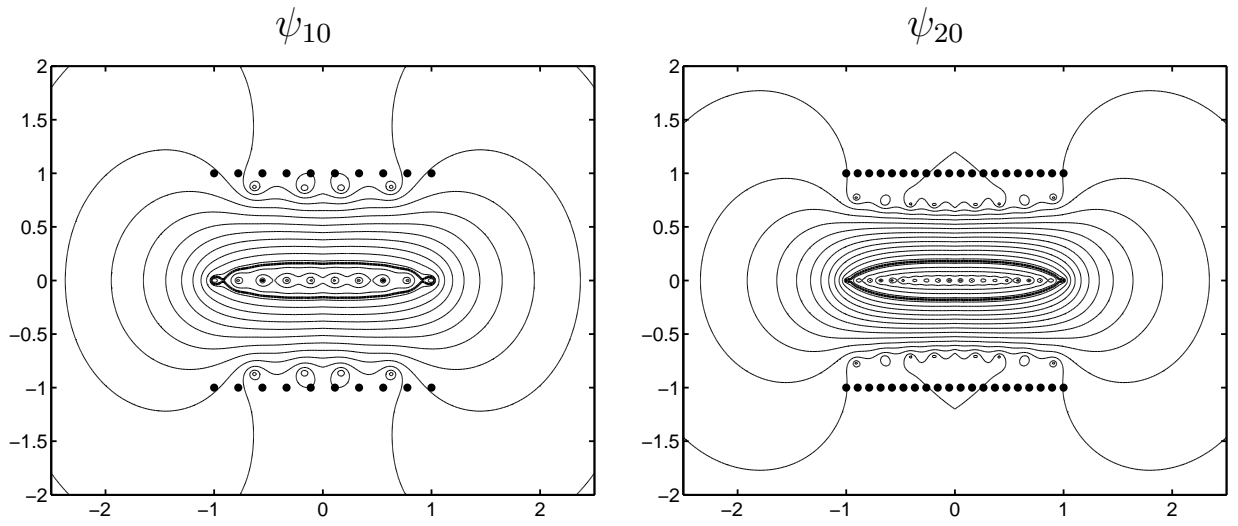

Figure 9: Contour levels of $\psi_{N}$ for multiquadric RBFs with equispaced centers and $\varepsilon=1$. Twenty contour levels are shown for $N=10$ and $N=20$. A thick contour marks the zero-level and the branch points of the basis functions are marked with dots. Other level values of $\psi_{20}$ shown are $-0.77,-0.71, \ldots, 0.25,0.31$.

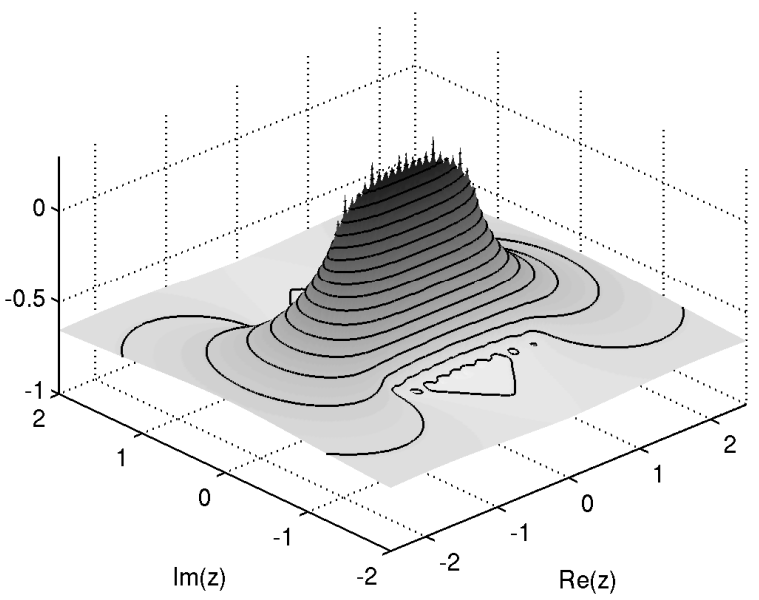

Figure 10: Surface plot of $\psi_{22}$ for multiquadrics with $\varepsilon=1$ and equispaced nodes. 

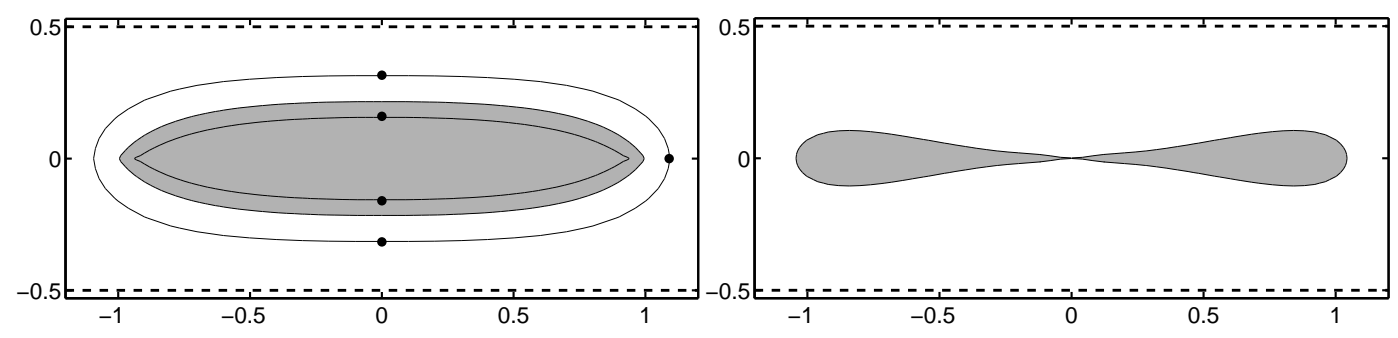

Figure 11: Estimated Runge regions (gray area) for inverse quadratic RBFs with $\varepsilon=1$, for uniform (left) and Chebyshev (right) node distributions. In the left frame, dots mark singularity locations of $f_{1}(x)=1 /\left(1+10 x^{2}\right), f_{2}(x)=1 /\left(1+36 x^{2}\right)$ and $f_{3}(x)=$ $\sqrt{1.09-x}$. Level curves passing at these locations are approximately $\psi_{\infty}(z)=-0.156$ and $\psi_{\infty}(z)=0.085$.

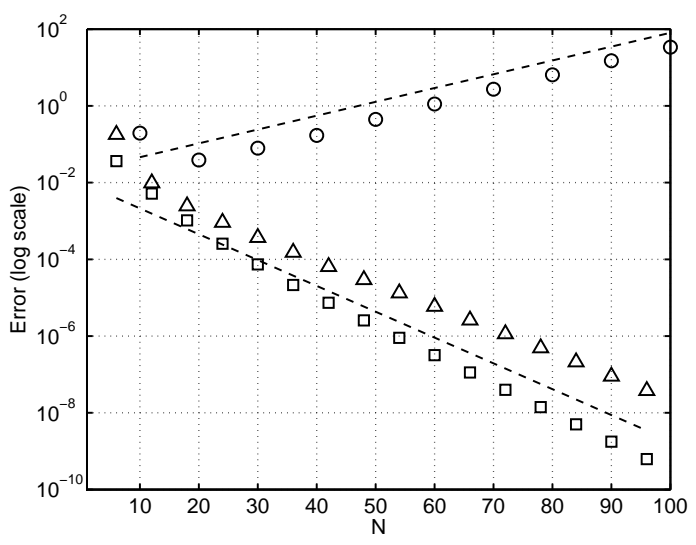

Figure 12: Maximum error in $[-1,1]$ in the computation of the interpolants of $f_{1}(\square), f_{2}$ $(\circ)$ and $f_{3}(\triangle)$. The estimates $O\left(e^{-0.156 N}\right)$ and $O\left(e^{0.085 N}\right)$ are shown with dashed lines.

three test functions, $f_{1}(x)=1 /\left(1+10 x^{2}\right), f_{2}(x)=1 /\left(1+36 x^{2}\right)$ and $f_{3}(x)=\sqrt{1.09-x}$, together with estimated values of the contours of $\psi_{\infty}(z)$ passing through these points. According to Corollary 4A, the interpolants of $f_{1}$ and $f_{3}$ should converge at similar rates, as these functions have analytic extensions up to (approximately) the same equiconvergence contour. Interpolants of $f_{2}$, on the other hand, are predicted to diverge exponentially with $N$ by Corollary $2 \mathrm{~A}$.

In Fig. 12 the computed errors in the interpolation of $f_{1}, f_{2}$, and $f_{3}$ are compared to the estimated convergence rates given by the equiconvergence contours shown in Fig. 11 and show very good agreement. In particular, the divergence of interpolants of $f_{2}$ is evident, and as expected, the rates of convergence for $f_{1}$ and $f_{3}$ are nearly the same. As in polynomial interpolation, we also observe that when interoplants blowup, they do so outside the contour passing through the singularities of the target function, as illustrated in Fig. 13. At present, however, we are unable to prove that this must happen in general. 


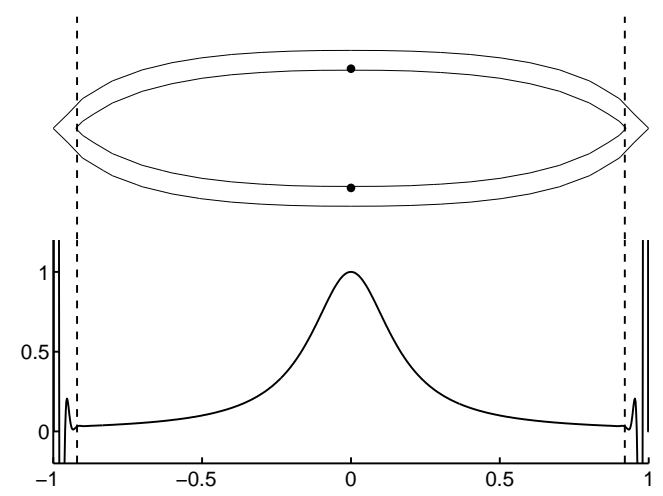

Figure 13: Inverse quadratic interpolant of $f_{2}$ on equidistant points. At the top of this figure, a plot of the Runge region and the level curve of $\psi_{\infty}$ passing through the pole locations of $f 2$ is shown.

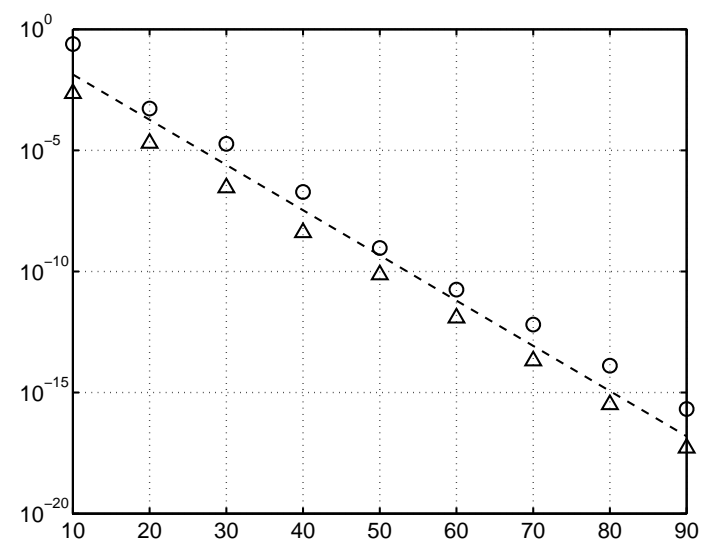

Figure 14: Maximum error in $[-1,1]$ in the interpolation of $f_{4}(x)=\sin (10 x)$ (o) and $f_{5}(x)=1 /\left(1+4 x^{2}\right)(\triangle)$. The estimate $O\left(e^{-0.43 N}\right)$ is shown with a dashed line.

Another important observation is that $\psi_{\infty}$ seems to be constant outside the strip $|\operatorname{Im}(z)|=1 /(2 \varepsilon)$, indicating that for functions that are analytic inside the strip, convergence rates are independent of singularity locations. Fig. 14 present the maximum computed error in the interpolation of two fairly different functions: $f_{4}(x)=\sin (10 x)$ and $f_{5}(x)=1 /\left(1+4 x^{2}\right)$. The former is entire, while the latter has poles at $\pm i / 2$. The convergence rates of inverse quadratic interpolants, however, are about the same as suggested by our estimate of $\psi_{\infty}$ in Fig. 7 .

\section{Good nodes for interpolation}

Unfortunately, as pointed out in Proposition 5, Lebesgue constants $\Lambda_{N}$ grow at exponential rates when $\psi(z)>0$ in a neighborhood of the unit interval. Both uniform 


\begin{tabular}{|c|c|c|c|c|c|c|}
\hline & \multicolumn{3}{|c|}{$\varepsilon=0.5$} & \multicolumn{3}{|c|}{$\varepsilon=1$} \\
\hline$N$ & $X^{\text {unif }}$ & $X^{\text {cheb }}$ & $X_{0.8}^{k t e}$ & $X^{\text {unif }}$ & $X^{\text {cheb }}$ & $X_{0.9}^{k t e}$ \\
\hline 10 & 6.8 & 3.5 & 2.3 & 1.8 & 4.8 & 2.4 \\
\hline 20 & $4.5 \mathrm{e}+2$ & 7.5 & 2.7 & $2.5 \mathrm{e}+1$ & $2.6 \mathrm{e}+1$ & 3.1 \\
\hline 30 & $5.9 \mathrm{e}+4$ & $1.8 \mathrm{e}+1$ & 2.9 & $5.0 \mathrm{e}+2$ & $2.2 \mathrm{e}+2$ & 3.7 \\
\hline 40 & $9.2 \mathrm{e}+6$ & $5.0 \mathrm{e}+1$ & 3.0 & $1.2 \mathrm{e}+4$ & $2.2 \mathrm{e}+3$ & 4.2 \\
\hline 50 & $1.7 \mathrm{e}+9$ & $1.6 \mathrm{e}+2$ & 3.1 & $3.5 \mathrm{e}+5$ & $2.2 \mathrm{e}+4$ & 4.7 \\
\hline 60 & $2.9 \mathrm{e}+11$ & $5.1 \mathrm{e}+2$ & 3.3 & $1.0 \mathrm{e}+7$ & $2.4 \mathrm{e}+5$ & 5.4 \\
\hline
\end{tabular}

Table 1: Estimated Lebesgue constants $\Lambda_{N}$ for inverse quadratic RBFs with $\varepsilon=0.5$ and $\varepsilon=1$. Three node distributions are considered in each case: uniform, Chebyshev, and mapped Chebyshev.

and Chebyshev node distributions therefore lead to large Lebesgue constants and instabilities. As in polynomial interpolation, we can prevent the rapid growth of $\Lambda_{N}$ by interpolating at near-best nodes. Unfortunately, analytic expressions for these nodes are not known and they need to be computed for each RBF and shape parameter. In (De Marchi et al., 2005), greedy algorithms were proposed for the computation of nearoptimal nodes. In this article, however, we explore mapped Chebyshev points as an alternative.

Kosloff \& Tal-Ezer (1993) have introduced the family of nodes

$$
x_{j}^{k t e(\alpha)}:=\arcsin \left(\alpha x_{j}^{c h e b}\right) / \arcsin (\alpha), \quad j=1, \ldots, N,
$$

where $x_{j}^{\text {cheb }}$ is a Chebyshev node. Other mappings have been proposed in Hale \& Trefethen (2008) and there is a close connection between RBFs and mapped polynomials. We do not explore this connection beyond node distributions in this article. The mapped nodes in (7.1) are equally spaced when $\alpha=1$ and approach Chebyshev nodes as $\alpha \rightarrow 0$. Instead of solving an $N$-parameter optimization problem to find optimal nodes for interpolation, we optimize the parameter $\alpha$ in (7.1) in order to minimize the Lebesgue constant.

Tabel 1 presents approximate Lebesgue constants for equispaced, Chebyshev and mapped nodes. For inverse quadratic RBFs with $\varepsilon=0.5$ the optimal value of $\alpha$ is approximately 0.8 , while for $\varepsilon=1$, the optimal value is nearly 0.9 . The numbers shown in this table indicate that these nodes give much smaller Lebesgue constants than either Chebyshev or equispaced nodes. In fact, all values shown are of order 1 for mapped nodes, while the exponential growth of $\Lambda_{N}$ can be observed for the other node distributions.

Figure 15 shows the level curves of $\psi_{30}$ and $\psi_{70}$ for inverse quadratics with $\varepsilon=1$ and mapped nodes with $\alpha=0.9$. In this case, the level curves all correspond to negative values of $\psi_{N}$ which gives further indication that this simple choice of nodes is effective in preventing the Runge phenomenon. Moreover, the convergence rates estimated by these levels are significantly better than those in Fig. 7 and Fig. 8. The better convergence 

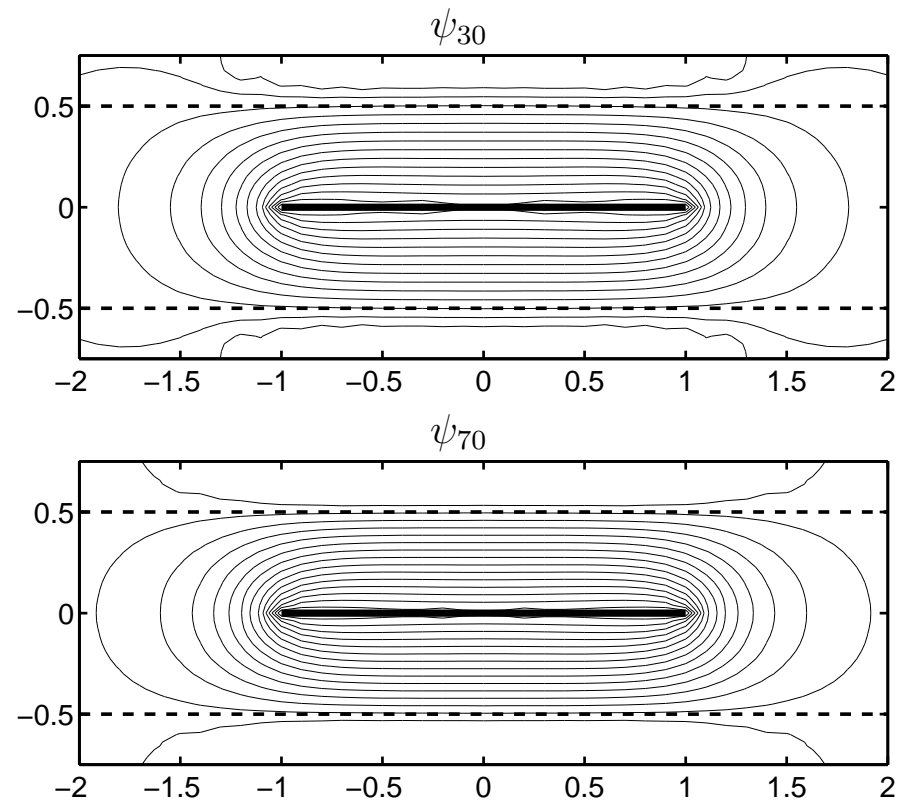

Figure 15: Contour levels of $\psi_{N}$ for inverse quadratic RBFs, mapped Chebyshev nodes with $\alpha=0.9$, and $\varepsilon=1$. Fifteen contour levels are shown for $N=30$ and $N=70$. A thick line marks the unit interval and dashed lines represent $|\operatorname{Im}(z)|=1 /(2 \varepsilon)$. Level curves in the last frame correspond to $\psi_{70}=-0.65,-0.60, \ldots,-0.06,-0.01$.

rates are confirmed in Fig. 16, where the error is presented for $f_{1}(x)=1 /\left(1+36 x^{2}\right)$ and $f_{4}(x)=\sin (10 x)$.

\section{Concluding remarks}

The question in the title has been answered using the contour error formula in Theorem 2, from which error bounds have been derived in Corollary $2 \mathrm{~B}$ - a general answer to a general question. In specific cases we provide detailed results. Theorem 3 states that inverse quadratic RBFs converge to functions that are analytic inside the strip $|\operatorname{Im}(z)|<1 /(2 \varepsilon)$ at exponential rates, and the rates of convergence are estimated in Section 5 for two values of the shape parameter and node distributions. Theorem 4 states that Gaussians RBF interpolants on equally spaced nodes behave asymptotically as polynomials. Gaussians and polynomials are entire functions. Both bases allow for super-geometric rates of convergence for very smooth functions. Inverse quadratics and multiquadircs, on the other hand, have singularities which approach the real line as the shape parameter $\varepsilon$ is increased, and their behavior can be compared to mapped polynomial and rational approximations. Although special attention has been given to inverse quadratics and Gaussians, we believe our results provide good insight into the convergence of smooth RBF interpolants in general.

The theory of convergence of linear methods to analytic functions in the complex 


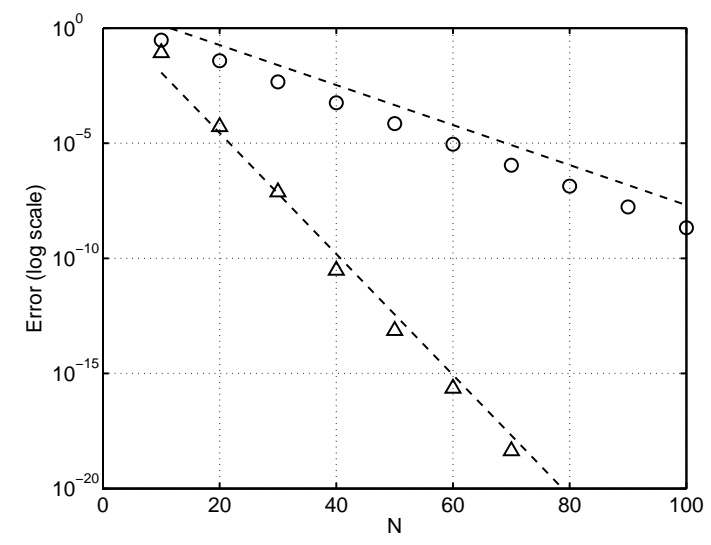

Figure 16: Maximum error in $[-1,1]$ in the interpolation of $f_{1}(\circ)$ and $f_{4}(\triangle)$. The estimates $O\left(e^{-0.2 N}\right)$ and $O\left(e^{-0.65 N}\right)$ are shown with dashed lines.

plane is rich, but has been ignored to a large extent in the literature on RBF methods. The general form of the error presented in Corollary 2A and error bound in Corollary 2B provide a general framework for comparing the convergence of different methods, including their susceptibility to the Runge phenomenon. In Section 5 equiconvergence contours were estimated. Numerical experiments and the predicted convergence rates showed very good agreement. As previously pointed by Platte \& Driscoll (2005), Fornberg \& Zuev (2007) and Schaback (2008), standard RBF methods based on interpolation are often unstable when smooth basis functions are used to recover functions from scattered data due to large Lebesgue constants. One may prevent the exponential growth of Lebesgue constants by using near-optimal point locations as demonstrated in Section 5.

Only interpolation with fixed shape parameter has been considered in this work. However, the framework provided by the Hermite-type error formula can be used to study the convergence of least-squares (Platte \& Driscoll, 2006) and variable shape parameters approximations. The results presented here give further evidence that the use of variable shape parameters may be required for the effective recovery of smooth functions, specially on multi-dimensions, where near optimal nodes are expensive to compute.

\section{Acknowledgments}

The author is grateful for advice from Bengt Fornberg and Nick Trefethen. This work was funded by UK EPSRC grant EP/E045847/1.

\section{Appendix: proof of Theorem 4}

The proof requires the following result which has been derived from Lemma 2.1 in (Platte \& Driscoll, 2005). 
Lemma 6 Suppose $f$ is analytic in a bounded closed simply connected region $R$ and $C$ is a simple, closed, rectifiable curve that lies in $R$ and contains the interpolation points $x_{j}=-1+2(j-1) /(N-1), j=1, \ldots, N$. Then, for sufficiently large $N$, the remainder of the Gaussian RBF interpolation for $f$ at $x$ can be represented as the contour integral

$$
f(x)-S_{f, N}(x)=\frac{\beta \eta_{N}(x)}{2 \pi i} \int_{C} \frac{f(z) e^{\beta z}}{\eta_{N}(z)\left(e^{\beta z}-e^{\beta x}\right)} d z
$$

where $\eta_{N}(x)=e^{-\varepsilon^{2}(x+1)^{2}} \prod_{k=1}^{N}\left(e^{\beta x}-e^{\beta x_{k}}\right)$ and $\beta=\frac{\varepsilon^{2} 4}{N-1}$.

We can rewrite (8.1) as

$$
\left(f(x)-S_{f, N}(x)\right) e^{\varepsilon^{2}(x+1)^{2}}=\frac{1}{2 \pi i} \int_{C} \frac{g(z)}{z-x} \mathcal{R}_{N}(z, x) d z,
$$

where $g(z)=e^{\varepsilon^{2}(z+1)^{2}} f(z)$ and

$$
\mathcal{R}_{N}(z, x)=\beta e^{\beta z} \frac{(z-x) \prod_{k=1}^{N}\left(e^{\beta x}-e^{\beta x_{k}}\right)}{\left(e^{\beta z}-e^{\beta x}\right) \prod_{k=1}^{N}\left(e^{\beta z}-e^{\beta x_{k}}\right)} .
$$

Therefore,

$\log \left|\mathcal{R}_{N}(z, x)\right|=\log \beta+\beta \operatorname{Re}(Z)+\log |z-x|-\log \left|e^{\beta z}-e^{\beta x}\right|+\sum_{k=1}^{N} \log \left|e^{\beta x}-e^{\beta x_{k}}\right|-\sum_{k=1}^{N} \log \left|e^{\beta z}-e^{\beta x_{k}}\right|$ and for $z \neq x$,

$$
\begin{aligned}
\lim _{N \rightarrow \infty} \frac{1}{N} \log \left|\mathcal{R}_{N}(z, x)\right| & =\lim _{N \rightarrow \infty} \frac{1}{N} \sum_{k=1}^{N} \log \left|e^{\beta x}-e^{\beta x_{k}}\right|-\frac{1}{N} \sum_{k=1}^{N} \log \left|e^{\beta z}-e^{\beta x_{k}}\right| \\
& =u(x)-u(z),
\end{aligned}
$$

where

$$
u(z)=\frac{1}{2} \int_{-1}^{1} \log |z-x| d x=-1+\frac{1}{2} \operatorname{Re}((z+1) \log (z+1)-(z-1) \log (z-1)) .
$$

To complete the proof, we note that

$$
\max _{x \in[-1,1]} u(x)-u(z)
$$

is positive whenever $z$ is inside the region enclosed by

$$
2 \log 2=\operatorname{Re}((z+1) \log (z+1)-(z-1) \log (z-1))
$$

and negative outside. 


\section{References}

J.-P. Berrut \& L. N. Trefethen (2004). 'Barycentric Lagrange interpolation'. SIAM Rev. 46(3):501-517.

J. P. Boyd (2009). 'Error saturation in Gaussian radial basis function on a finite interval'. Preprint.

M. D. Buhmann (2003). Radial Basis Functions: Theory and Implementations. Cambridge University Press, Cambridge.

P. J. Davis (1975). Interpolation and Approximation. Dover Publications Inc., New York.

S. De Marchi, et al. (2005). 'Near-optimal data-independent point locations for radial basis function interpolation'. Adv. Comput. Math. 23(3):317-330.

J. D. Donaldson \& D. Elliott (1972). 'A Unified Approach to Quadrature Rules with Asymptotic Estimates of Their Remainders'. SIAM Journal on Numerical Analysis 9(4):573-602.

T. A. Driscoll \& B. Fornberg (2002). 'Interpolation in the limit of increasingly flat radial basis functions'. Comput. Math. Appl. 43(3-5):413-422.

G. E. Fasshauer (2007). Meshfree Approximation Methods with MATLAB. World Scientific Publishing Co. Pte. Ltd., Hackensack, NJ.

B. Fornberg (1996). A Practical Guide to Pseudospectral Methods. Cambridge University Press, Cambridge.

B. Fornberg \& J. Zuev (2007). 'The Runge phenomenon and spatially variable shape parameters in RBF interpolation'. Comput. Math. Appl. 54(3):379-398.

N. Hale \& L. N. Trefethen (2008). 'New quadrature methods from conformal maps'. SIAM J. Numer. Anal. 46(2):930-948.

D. Kosloff \& H. Tal-Ezer (1993). 'A modified Chebyshev pseudospectral method with an $O\left(N^{-1}\right)$ time step restriction'. J. Comput. Phys. 104(2):457-469.

W. R. Madych \& S. A. Nelson (1992). 'Bounds on multivariate polynomials and exponential error estimates for multiquadric interpolation'. J. Approx. Theory 70(1):94-114.

R. B. Platte \& T. A. Driscoll (2005). 'Polynomials and potential theory for Gaussian radial basis function interpolation'. SIAM J. Numer. Anal. 43(2):750-766.

R. B. Platte \& T. A. Driscoll (2006). 'Eigenvalue stability of radial basis function discretizations for time-dependent problems'. Comput. Math. Appl. 51(8):1251-1268. 
R. B. Platte \& A. Gelb (2009). 'A hybrid Fourier-Chebyshev method for partial differential equations'. J. Sci. Comput. 39(2):244-264.

C. Runge (1901). 'Über empirische Funktionen and die Interpolation zwischen äquidistanten Ordinaten'. Z. Math. Phys. 46:224-243.

R. Schaback (2008). 'Limit problems for interpolation by analytic radial basis functions'. J. Comput. Appl. Math. 212(2):127-149.

L. N. Trefethen (2008). 'Is Gauss quadrature better than Clenshaw-Curtis?'. SIAM Rev. 50(1):67-87.

A. H. Turetskii (1940). 'The bounding of polynomials prescribed at equally distributed points'. Proc. Pedag. Inst. Vitebsk 3:117-127. Russian.

J. A. C. Weideman \& L. N. Trefethen (2007). 'The kink phenomenon in Fejér and Clenshaw-Curtis quadrature'. Numer. Math. 107(4):707-727.

H. Wendland (2005). Scattered Data Approximation. Cambridge University Press, Cambridge. 